\title{
Improvement for IEEE 802.11 PCF Access Mechanism
}

\author{
Ying,Huang ${ }^{1, a}$, Lin Zhou ${ }^{2,{ }^{*} b}$ and Xin, $\mathrm{Li}^{3, \mathrm{c}}$ \\ ${ }^{1}$ Faculty of Information Engineering, China University of Geosciences, CN \\ 2 Faculty of Information Engineering, China University of Geosciences, CN \\ ${ }^{3}$ Deparment of Information Engineering, China University of Geosciences, CN \\ ahying@cug.edu.cn, ${ }^{\mathrm{b}}$ 20890590@qq.com, ${ }^{\mathrm{c}} 15051249 @ q q . c o m$ \\ * the corresponding author
}

Keywords: IEEE 802.11; Media Access Control. PCF; DCF

Abstract. Nowadays, it is a popular life trend to surf on the Internet, make a phone call and watch video by the portable terminals anytime and anywhere. Especially at the crowded commercial areas, exhibition halls, airports, the number of users are a lot but the wireless access points APs is limited, the traffic of video and audio through WLAN increases sharply. This kind of traffic has high performance requirements on the end-to-end delay of network.. This paper proposed a new improved access scheme in order to solve the real-time performance insufficient of transmission traffic when multiple access through the PCF protocol. The improved access scheme changed the polling and sorting of PCF to the competition and sorting.This effectively reduced the end-to-end delay of network ,ensured that the transmission delay can meet the requirements of real-time streaming and improved the transmission quality of the real-time streaming. This paper also compared the performance of several mechanisms of IEEE 802.11 MAC. Simulation results illuminates that the improved mechanism is able to increase throughput of the Internet as well as keep a comparatively short delay of end-to-end connection.

\section{Introduction}

With the social progress and economic development, the intelligent terminals such as mobile phone, PDA and so on have become very popular. Wireless network become the focus of people chase with its "ubiquitous" charm. Currently, it is a popular life trend to surf on the Internet, make a phone call and watch video by the portable terminals anytime and anywhere. According to statistics, the number of the global sold WLAN terminals was 934 million in 2013. Among these terminals, the smartphone accounted for $73 \%$, the tablet PC accounted for $12 \%$, the notebook accounted for $10 \%$. Someone say that by the end of 2016, more than half of the Internet traffic will come from the wi-fi connection, and the traffic of network video and audio will greatly increase. This kind of traffic has high performance requirements on the end-to-end delay of network. Especially at the crowded public areas, the number of mobile sites with a AP wireless access point increases greatly. How to improve the access performance with a lot sites? The access technology need to be improved. It is worth deep studying that how to ensure the network transmission performance of real-time traffic.

This paper proposed a new improved access scheme in order to solve the real-time performance insufficient of system transmission traffic when multiple access through the PCF protocol. The improved access scheme changed the polling and sorting of PCF to competition and sorting. This effectively reduced the end-to-end delay of network, ensured that the transmission delay can meet the requirements of real-time streaming and improved the transmission quality of the real-time streaming.

This paper is divided into five sections to discuss the problem. The first section introduced the application situation of WLAN access and present its existing problems technologically; The second section reviewed the work of 802.11 MAC Protocol. The third section described our proposal; The fourth section check our solution by simulation experiment and analyzed the simulation results. The firth section is the conclusion. 


\section{IEEE 802.11 Wireless LAN MAC Protocol}

\section{Work of 802.11 MAC Protocol}

MAC layer of 802.11 determines the wireless sites in BSS when to send or receive data by the coordination function. MAC layer includes two sub-layers: distribute coordination function(DCF) and point coordination function(PCF) . DCF doesn't use center control, but using distributed access algorithm based on CSMA mechanism in each site, lets each site get the send right through channel competition. The 802.11 protocol says that all sites must have DCF. PCF takes dynamic controlled access. It gets the node channel access opportunities according to a certain regulation control specified by network. The different nodes orderly access channel to send data by the arrangements of the specific regulation, that is called Point Coordination Function(PCF) in IEEE 802.11 protocol[1][4][5].

\section{PCF Work Mode of CSMA/CA Protocol}

The basic principle of PCF is using Point Coordinator (PC) to poll nodes, centralized control the access of media . PCF can only be applied to WLAN with infrastructure, usually the Access Point (AP) serves as the Point Coordinator. PCF takes access algorithm under centralized control, uses AP to centralized control the activities in BSS, with the method similar to explore to give each stite the right of sending data in turn. This avoids a collision. PCF is suited for streaming transmission and real-time data transmission which is sensitive to time delay .

\section{Problems of PCF}

PCF protocols provides an uncontested service, each site wants to send data, so it has large influence on the transmission efficiency that how to determine the data sending priority of each site. At the same time, the AP needs to wait for a certain time after sending inquiry frame, the change of the time also has great influence on the transmission efficiency.

\section{Existing Improvements on PCF Work Mode}

About polling scheduling algorithm in PCF ,there are several scheduling algorithms:1.Round- Robin Scheme(R- Poll);2.Cyclic Shift Polling Scheme(CS- Poll);3.First- In- First- Out Polling Scheme(FIFO);4.Priority Polling Scheme(Priority);5.Contention Period Multipolling(CP- MP). Here, we study an uplink AWFQ algorithm (Station to AP) . In this algorithm, each site is divided into three different kinds of state to be transmitted. The three states are:Backlogged state, Unbacklogged state and Bad Channel state. The site will convert to a different state when in different case, all the link form a finite state machine[1][3][6][7][8][9].

By research and analysis, the traditional PCF scheduling algorithms such as a Round - Robin and FIFO algorithm take address polling, and their performance is easily affected by transmission type; About QoS, the Priority algorithm provides simple way to support QoS transmission, and can endure mild link error; About packet loss rate, CS - Poll is worse than $\mathrm{R}$ - Poll.

\section{Our Improvement Scheme}

\section{PCF Access Scheme with Competition Mechanism}

According to the above analysis, there mainly are two kinds of PCF scheduling algorithm. One is polling in turn, such as Round- Robin Scheme(R- Poll),Cyclic Shift Polling Scheme(CS- Poll),First- In- First- Out Polling Scheme(FIFO). These algorithms dispose every site. They waste time a lot when there are a great quantity of sites. The other is polling by priority .It is good for the users with a priority, does not dispose most sites. Therefore, our idea is to introduce competition mechanism in polling phase, form a mechanism with combining competition and sorting. This scheme takes the order which AP sends to sites to manage, divides 
a working cycle into two periods: competition time and allocation time. In competition time, each site send request $\mathrm{T} 1$ to $\mathrm{AP}$;In allocate time, $\mathrm{AP}$ notifies each site to send data frames $\mathrm{T} 2$ by the request order.

Supposed there are N sites in BSS . First, in competition time, every site contest with sending right. The AP received RTS frame from the sites which want to send data and maintains a list which rank the sending data order of each site according RTS arriving time. The content items of the table is shown by Table 1 below . Second, in distribution time, every site send data in turn as the order of the list. AP informs the corresponding site to send data, if the sites which will not send data receive the AP notice messages, these sites will be given a mark that states the sites will not send data in the period $i$. When previous site finished sending a data frame, after waiting a SIFS, the site will send data frames, and needn't compete channel with other sites, if the destination site correctly received the data frames from the source site, after waiting a SIFS, sending a ACK frame to AP. After AP receives the ACK frame, it sends out a message in the channel, and notifies the next site to send data.

The whole flow chart of our scheme is referred to references[10].

Table 1 sequence of requesting sites

\begin{tabular}{|l|l|l|l|}
\hline Sequence Number & Source Site ID & Destination Site ID & $\begin{array}{l}\text { Its Sending Data } \\
\text { Length in Bytes }\end{array}$ \\
\hline
\end{tabular}

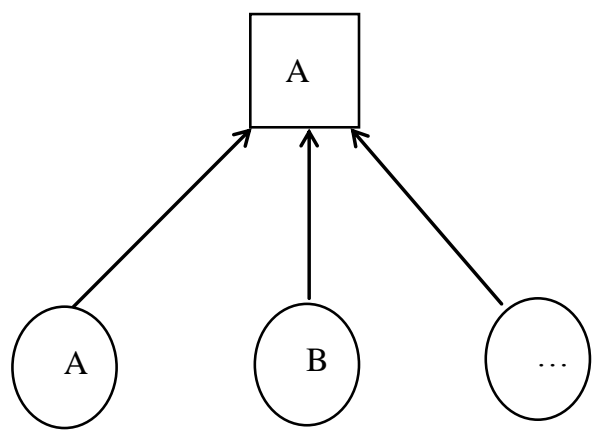

\begin{tabular}{|c|c|c|}
\hline Source & Destination & Duration \\
\hline A & $\mathrm{X}$ & $\mathrm{XXX}$ \\
\hline B & $\mathrm{Y}$ & $\mathrm{YYY}$ \\
\hline$\cdots$ & $\ldots$ & $\cdots \cdots$ \\
\hline
\end{tabular}

Sites with data frames send RTS to AP and AP maintain a list to record information according to the sequence of request.

Figure 1 sites send RTS to AP

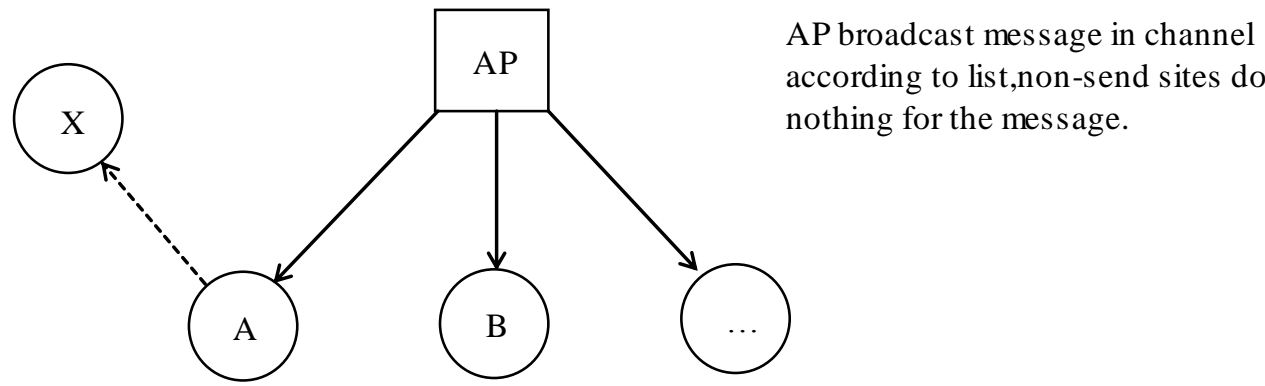

Figure 2 AP notifies the sites to send data

When two sites send RTS frames to AP at the same time, site monitoring channel discoveries that the channel state is from free to busy, there is a collision, Backoff algorithm will be executed. Backoff algorithm has slightly difference with binary exponential backoff algorithm. In binary exponential backoff algorithm, after the success of sending, backoff window reduces to the minimum; But the Backoff algorithm in the improvement scheme only minishes the backoff window to a half, ensures the conflict avoiding and fairness under high competition condition. 


\section{Eliminate Hidden Site and Exposed Site Problem}

Meanwhile, the improved scheme has solved hidden site and exposed site problem absolutely. The improved scheme used AP management channel. AP can communicate with each site when all the sites are within the scope of AP. When A site and the B site both want to send data to the site $\mathrm{C}$, according to the improved scheme, A and B should send request to AP (if request process has collision, the backoff algorithm will be executed, until the backoff time reduces to zero, the request can be sent again). Site sends RTS frame to AP, within the certain time, AP returns the CTS frame. Within the sending data period, the AP informs the site to send data according to the order in the list . Assuming that the site A sends request to AP prior to B, when the AP broadcast to channel, the site A sends data. The site B receiving broadcast message, according to the duration field in broadcast message, within the time T it will be marked as channel busy, don't send data to the site $\mathrm{C}$. After A finishes the sending, the AP will broadcast message to notify the site $\mathrm{B}$ to send data, thus solving the problem of hidden site.

Similarly, in exposed site problem, taking the improved scheme, the sending sites must send request to AP .According to the order of request, AP successively notifies the sites to send data, lets the receiving sites receive data successfully.

\section{Simulation and Analysis Based on OMNet++}

\section{Simulation Parameters}

In order to make a compare between our access scheme and the existing access schemes, we used the network simulation tools OmNet to simulate DFC access mechanism, PFC access mechanism and our access mechanism. The simulation mainly tested and compared their throughput, channel utilization and the end-to-end delay. The results were shown in the following charts.

\section{Simulation Results Analysis}

Throughput

\section{Throughput}

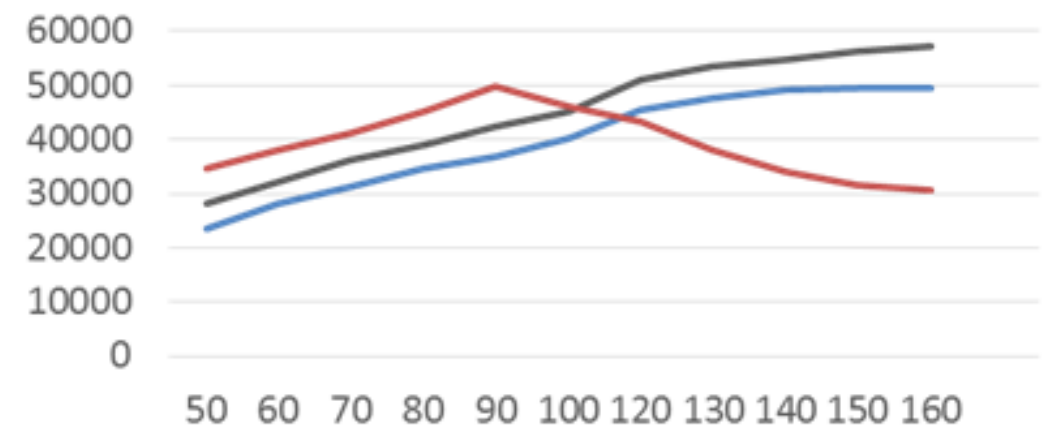

Site Number

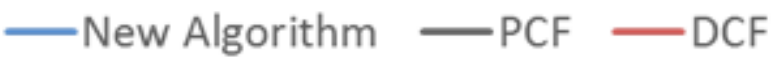

Figure 3 comparison of the throughput for different sites

The figure 3 shows the changes of the throughput between the AP control channel algorithm and DCF with the number of site changes. When the number of sites increased to a certain amount, the throughput of DCF drops rapidly, but the new algorithm can show the advantage with the site number increasing. When the amount of sites is large, the throughput of the new algorithm can be nearly maintained in stable constant. PCF is same as new algorithm. 


\section{Channel Utilization}

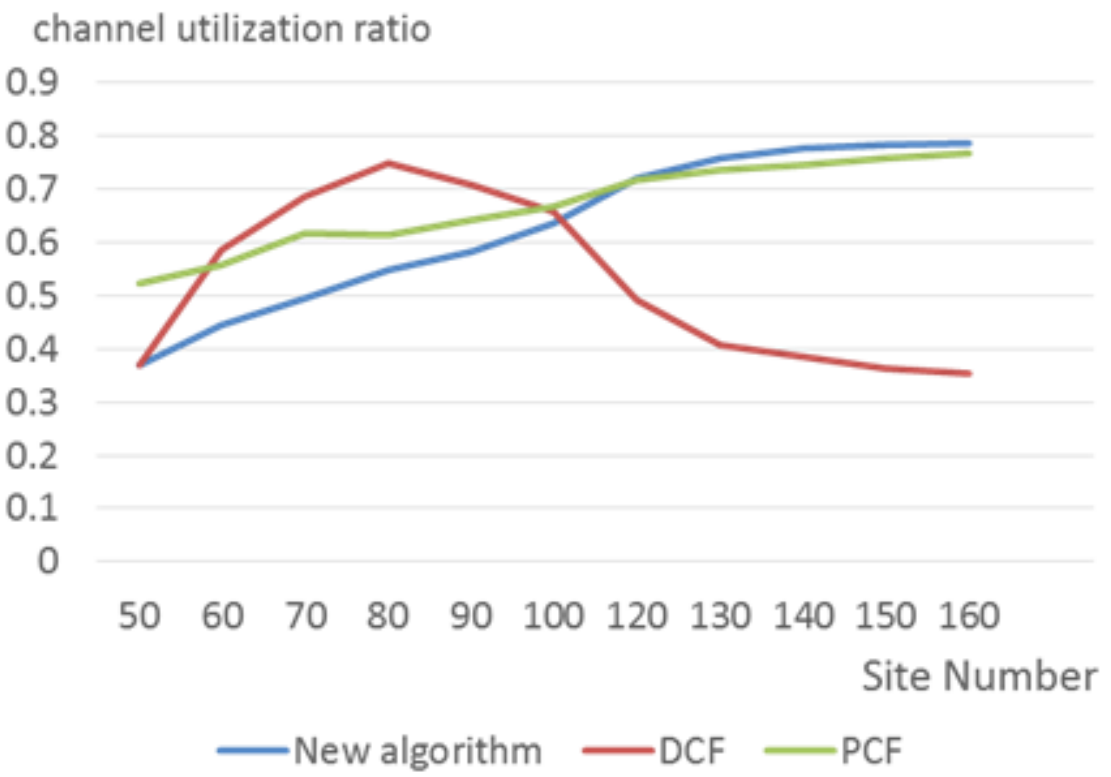

Figure 4 Channel Utilization Ratio for different numbers of sites

The figure 4 shows the channel utilization ratio compare of the three algorithms when the number of the sites is increasing. It can be seen when the number of access sites increases to a certain amount, the channel utilization of DCF decreases rapidly, but channel utilization of the new algorithm and PCF keeps stable. The main reason is when there are too many mobile sites in a service set, mobile sites want to send overmuch data, this causes many sites execute backoff algorithmat the same time, and the channel is free. There is no backoff time in the PCF and there is less than a half time of backoff in new algorithm.

4.2.3 End-to-End Delay

\section{End-to-End Delay}

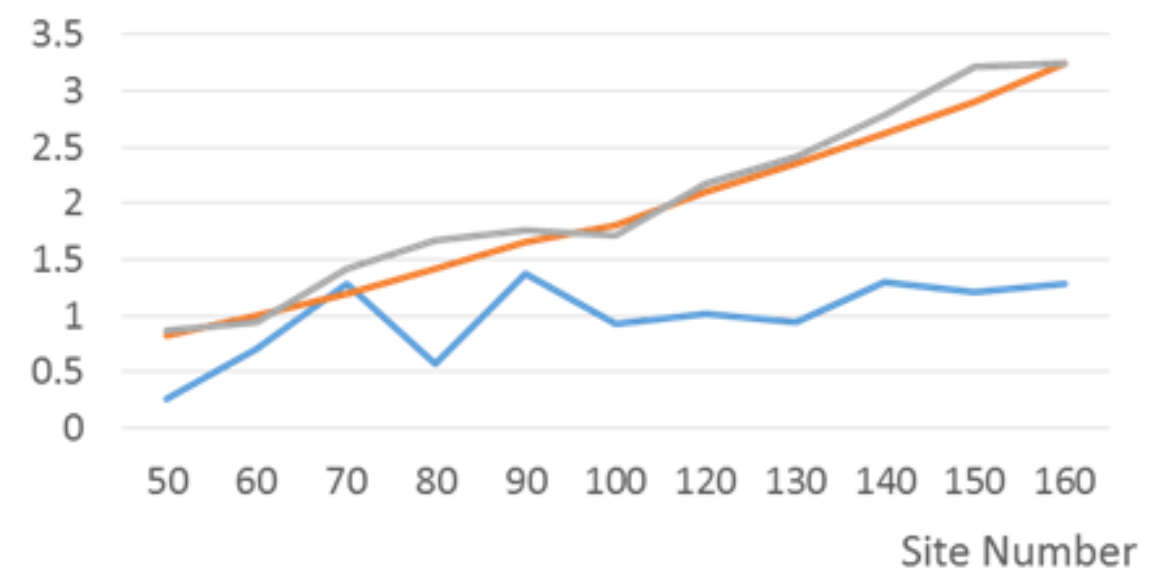

New Algorithm —PCF - DCF

Figure 5 End-to-end Delay

The figure 5 shows the end-to-end delay under different number of sites . Test results show that the end-to-end delay of the new mechanism is evident lower than the end-to-end delay of DCF and PCF. No matter whether the number of access site is many or few, the end-to-end delay of the new mechanism keeps stable and low, the more the access sites, the greater the gap,our mechanism has obvious advantage. According to our analyzation, since we eliminate the non request-to-send sites in the competitive phase, the end-to-end delay is largely reduced. 


\section{Conclusion}

Summarize the simulation results, we come to the result that our proposed new mechanism shows the distinct advantages in End-to-end Delay. Not only it has the very short end-to-end delay but also it has a high throughput and high ratio of channelutilization when the access sites are mass 。Without priority, the fairness of the scheme is also quite good. Especially our proposed new mechanism shows excellent performance for real traffic. The new mechanism is specifically conducive to audio and video transmission of demand for high quality of real-time performance.

In our future research, we will further analyze and confirm this improved mechanism, and we will design a more reliable simulation experimental model.

\section{References}

[1] Liao Young, Xu Chang Biao, Yang Shi Zhong. The Polling Mechanism in IEEE 802.11 PCF [J]. Microcomputer Information,2007,30:136-138.

[2] KE Chih-Heng, WEI Chih-Cheng, WU Tin-Yu. A Smart Exponential-threshold-linear Backoff Algorithm to Enhance the Performance of IEEE 802.11 DCF. Proc.IEEE 4th ChinaCOM Conference.2009

[3] Liao Yong. IEEE 802.11 Adaptive Polling Mechanism in PCF [D].Chongqing University,2007..

[4] Kwon Younggoo, Fang YuguangH,Latchman.A novel MAC protocol with fastcollision resolution for wireless LANs[A].Twenty-Second Annual Joint Conference of the IEEE Computer and Communications.2003

[5] Kang Kai, Lin Xiao Kang. A Method of Reducing Conflict in 802.11MAC IEEE layer [A]. Journal of Harbin Institute of Technology. 2009,41(1):156-160.

[6] Chen Xu Long, Peng Yu Xing. Research and Simulation of MAC Sub Layer DCF\&PCF IEEE802.11 [J]. Computer Engineering and Design,2008,05:1096-1099.

[7] Yan Xiao Dong, Wang Zhi Juan. An Improved PCF Protocol[J]. Journal of the Central University for Nationalities(NATURAL SCIENCE EDITION)),2008,02:44-47

[8] Ji Jing Chen. Research On WLAN PCF Algorithm Mechanism [D]. Chongqing University,2009.

[9] Shuai Xiao Ying, Qian Huan Yan. Real-time Access Control Protocol Based on PCF [J]. Journal of Nanjing University of Science and Technology,2012,02:266-271.

[10]Ying Huang,Aiwu Shi,Baoliang Hao,The Improved Research on IEEE 802.11 Mac Layer Access Mechanism. 2015 The $5^{\text {th }}$ International Workshop on Computer Science and Engineeering(WCSE2015) 